

\title{
MASS MATTERS:
}

\section{LATEST HIGGS BOSON RESULTS FROM THE LHC}

\section{The Higgs boson was discovered at the Large Hadron Collider in 2012. Since then, a comprehensive program has been ongoing to characterize it as precisely as possible. Can this particle help us solve some of the big open questions in physics?}

. Sarah Heim - DESY, Germany - DOI: https://doi.org/10.1051/epn/2021205

$\triangle$ Candidate event for a Higgs boson decay to two muons, produced together with two hadronic jets in the ATLAS detector.

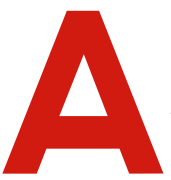
s particle physicists, our goal is to understand the fundamental building blocks of the universe by studying their properties and their interactions with each other. Our dream is to have a consistent and complete mathematical model of the elementary world, relying on as few input parameters as possible.

Our current best description of elementary particles and their interactions is called the Standard Model of particle physics. As an integral part of this model, the so-called Higgs mechanism describes a scalar field which permeates the universe, and gives mass to elementary particles by interacting with them. Furthermore, the quantum excitation of this field produces a scalar particle. In 2012, the ATLAS and CMS Collaborations at the
Large Hadron Collider (LHC) discovered such a particle, the Higgs boson.

The Standard Model predicts neither the Higgs boson mass nor the mass of the matter particles, the fermions, so these need to be measured. By now, the ATLAS and CMS experiments have determined the Higgs boson mass with a stunning accuracy of $1-2$ permille $[1,2]$. Knowing the masses, all other expected Higgs boson properties can be calculated, at least up to a certain precision. Measurements that do not agree with those predictions could point us towards a different mass-generation mechanism, perhaps as part of a theory that can explain some of the questions the Standard Model cannot answer, like the nature of dark matter or the origin of the matter-antimatter asymmetry in the universe. 


\section{Interacting with the Higgs boson}

Arguably the most interesting property of the Higgs boson is its interaction with other fundamental particles (and also with itself), as the strengths of these interactions are directly related to the particles' masses: the stronger the coupling, the larger the mass. The Standard Model does not explain why some particles, like electrons, have very small coupling strengths and are therefore extremely light, while others, like the top quark, weigh about as much as a gold atom. Measuring the strength of the Higgs boson interactions could possibly help us understand what lies behind the very different mass values found in the elementary world. The couplings between the Higgs boson and other particles can be extracted by measuring how exactly the Higgs boson is produced in the proton-proton collisions at the LHC and, since the Higgs boson has a very short lifetime, how it decays to other, lighter particles. Figure 1 shows the Standard Model predictions for Higgs boson production and decays.

The Higgs boson was discovered by filtering for collisions that result in two force-carrying bosons, in particular two photons or two $\mathrm{Z}$ bosons. Their invariant mass, i.e. the mass of a possible mother particle, was scanned in the search for an excess. In the years following the discovery, more data was collected and physicists in the ATLAS and CMS Collaborations continued to improve their analysis techniques, incorporating for example more advanced machine learning algorithms. This led to the discovery of Higgs boson decays to fermions, which are as hard to find as needles in a haystack due to many background processes that leave similar detector signatures. By now, we have discovered Higgs boson decays to pairs of tau leptons (the heaviest known leptons) and pairs of bottom quarks (the second heaviest known quarks). Higgs boson decays to top quarks are not allowed kinematically, because the top quark is heavier than the Higgs boson. Fortunately, the coupling to these heaviest of all known elementary particles can still be probed directly by disentangling different Higgs boson production mechanisms, in particular the production of a Higgs boson in association with two top quarks.

\section{Higgs boson production mechanisms}

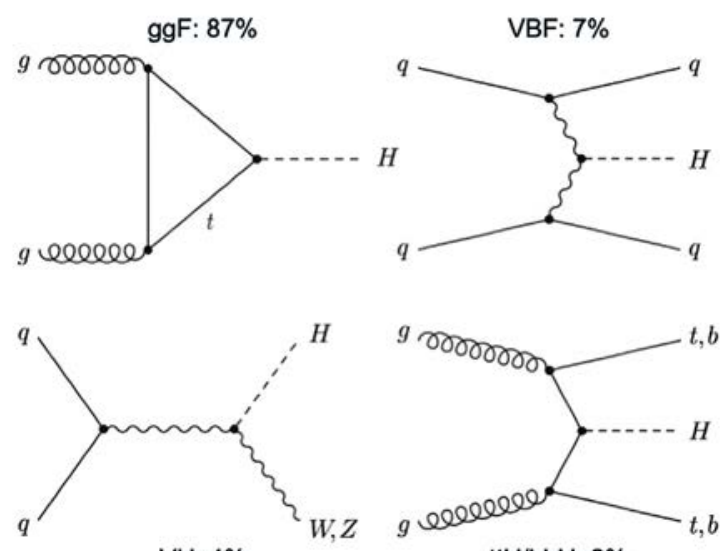

VH: $4 \%$
To understand whether all elementary particles receive their mass through the Higgs mechanism, it is now of utmost importance to probe the Higgs boson interactions also with lighter fermions. These couple more weakly to the Higgs boson, leading to extremely rare decays: For example, only 1 in 5000 Higgs bosons is expected to decay to two muons. It was possible last year to see first exciting hints of this decay at both the ATLAS and CMS experiments $[3,4]$. Thanks to the extremely strong magnetic field that allows the detector to determine the muon momentum with stunning precision, the CMS Collaboration found a signal consistent with the Higgs boson decaying to two muons, as shown in Figure 2, left. The significance of the signal is 3 sigma, which means the probability that it is due to a statistical fluctuation is less than 1 in 700 . The combination of CMS and ATLAS results would increase the significance well above 3 sigma, providing strong evidence for this decay. For a discovery, a probability of 1 in 3.5 Mio is required - which we aim to achieve with more proton-proton collision data.

The best way to get a complete picture of Higgs boson interactions with other particles is to statistically combine measurements of all accessible Higgs boson production mechanisms and decays. With the assumption that the Standard Model describes the general structure of the interactions (see below for tests of the symmetry behaviour), one can then compare the measured coupling strengths to the Standard Model predictions. Figure 2, right, shows the results and achieved precision from the CMS experiment for the Higgs boson couplings to different particles excellent agreement is found so far between all measurements and the Standard Model predictions $[5,6]$.

\section{The Higgs boson and dark matter}

One of the biggest puzzles in physics today is the question of what constitutes the observed dark matter in the universe. Dark matter particles, if they exist, must be massive, but only interact very weakly with normal matter. Physicists attempt to track down these elusive particles with multiple

\section{Higgs boson decays}

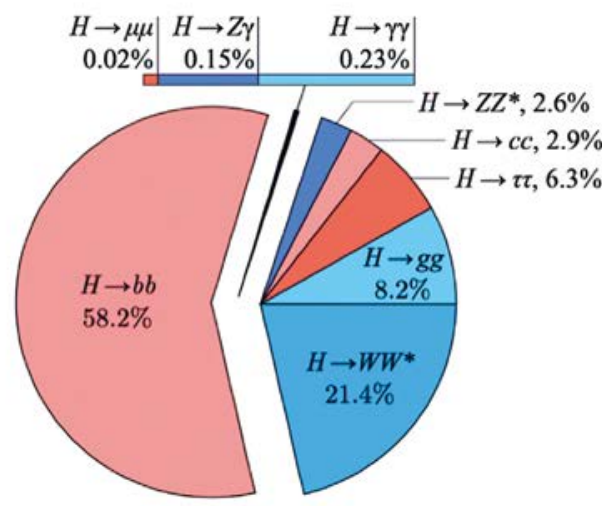

vFIG. 1: Left: Higgs boson production mechanisms in proton-proton collisions at $13 \mathrm{TeV}$, according to the Standard Model of particle physics, assuming a Higgs boson mass of $125 \mathrm{GeV}$. The production mechanisms, from top left, clockwise, are: gluon-gluon fusion, vector-boson fusion, associated production with two top (or two bottom) quarks, and associated production with a Z or W boson. Right: Standard Model predictions for the fractions of Higgs boson decays to different particles, for a Higgs boson with a mass of $125 \mathrm{GeV}$. The fractions of the decays to fermions are shaded in red, and the fractions of decays to bosons are shaded in blue. 


\footnotetext{
- FIG. 2: Left:

Reconstructed

invariant mass

distribution in

collision events with

two muons recorded

by the CMS detector.

The measured data

points are shown in

black. The events are

weighted according

to their probability

to contain a Higgs

boson decay to

two muons. In the

bottom panel, the

expected background

is subtracted and the

excess around the

Higgs boson mass is visible.

Right: Coupling strengths of

different elementary particles to the

Higgs boson from

CMS measurements, plotted vs the

respective particle's mass. The Standard Model prediction is the dashed line. The bottom panel shows

the ratio between measurements and prediction. Current measurement

uncertainties are

shown as error bars. It can be seen that the measurements agree

very well with the

linear prediction from the Standard Model.
}

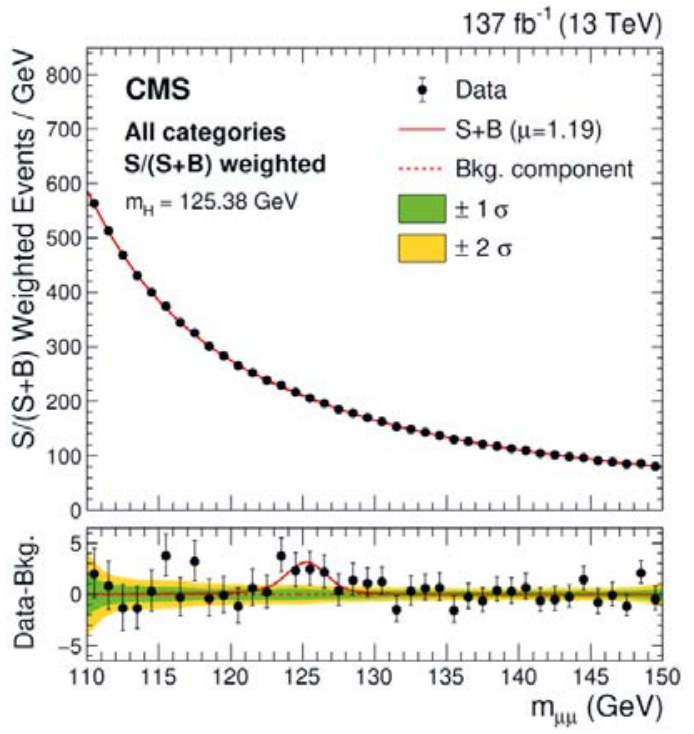

- - strategies: Trying to capture the extremely rare in teractions of dark matter in gigantic detector volumes, or through strong magnetic fields; looking for cosmic radiation that could come from dark matter decays somewhere in the universe; and trying to produce dark matter in accelerators.

Given that we know dark matter is massive, it makes sense to assume that it interacts with the Higgs boson. If the dark matter particles are in the right mass range, there is even the possibility to find them in decays of the Higgs boson. Since dark matter does not interact with the ATLAS or CMS detectors, we search for these decays by selecting collisions with a momentum imbalance, where detected particles recoil against something invisible.

At both LHC experiments, no hint of Higgs bosons decaying to dark matter has been observed $[7,8]$. Combining a number of analyses with different search strategies, a recent ATLAS analysis managed to constrain the fraction of Higgs bosons decaying to dark matter to smaller than $11 \%$, an impressive achievement, which nonetheless leaves plenty of space for the discovery of such decays in the future.

\section{Is the Higgs boson symmetric?}

The Standard Model predicts that the Higgs boson interactions with other particles should not change under various symmetries, in particular the case where the space coordinates are flipped (mirror symmetry) and the charges of the interacting particles are swapped simultaneously (for example a negative tau lepton vs its positive antiparticle). This symmetry is called Charge-Parity (CP) symmetry, and if it is fulfilled, as the Standard Model predicts, the Higgs boson coupling is called CP-even. Beyond the Standard Model, a coupling could also be CP-odd or a mixture containing an even and odd component.

For Higgs boson interactions with force-carrying bosons, it was already shown that the CP symmetry holds to a large extent. Last year, ATLAS and CMS also tested the symmetry properties of the Higgs boson coupling to top quarks and tau leptons $[9,10,11]$. In both cases, the

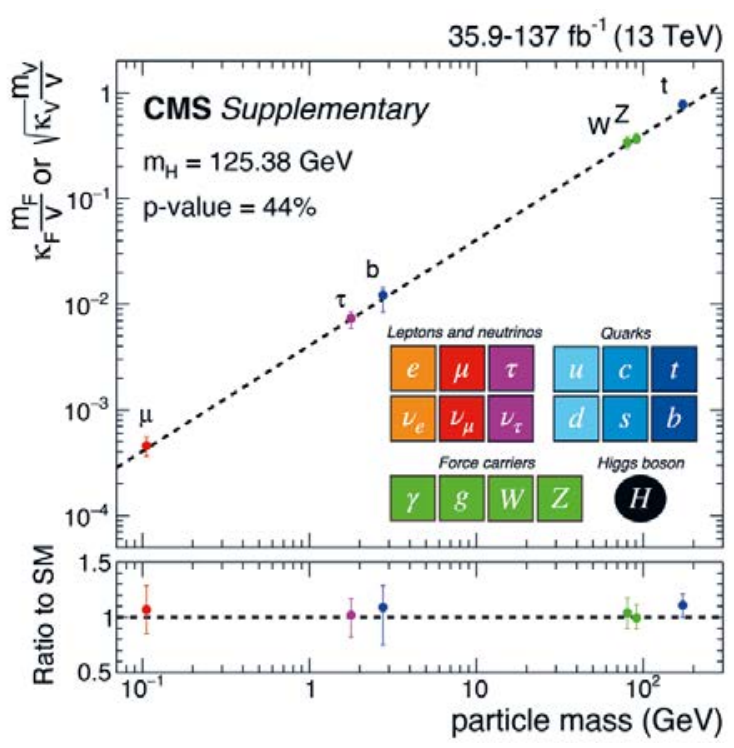

data clearly favors the CP-even over the CP-odd hypothesis with a significance of more than 3 sigma. Large CPodd admixtures are excluded as well. Other checks of the Higgs boson coupling structures have so far also solidly confirmed the Standard Model predictions.

\section{Extending the Higgs family}

Given all the successes and shortcomings of the Standard Model, it is natural that for a long time physicists have tried to find extensions to this model. Many of the new models predict more than one Higgs boson. In fact, one of the most popular classes of models, supersymmetry, states that there are at least five Higgs bosons, two of which are charged, and one of which violates CP symmetry. The additional Higgs bosons could be lighter or heavier than the Higgs boson we already found.

At the LHC, an intensive search is ongoing for additional Higgs bosons, similar to the original Higgs boson search. Furthermore, the interactions of the already-discovered Higgs boson could be affected by their existence; this means that precise measurements of Higgs boson properties - especially interaction strengths and structures - are also crucial in these investigations. In fact, it turns out that the sensitivity of the two approaches (search and measurement) is quite complementary, and, since nothing new has been found so far, they can exclude different areas of model parameter space, telling us at least where we do not have to search anymore [12].

\section{Characterizing the Higgs boson - Is that it?}

The landscape of Higgs boson physics remains interesting: Both ATLAS and CMS are still measuring Higgs boson properties and searching for additional Higgs bosons with the data set recorded between 2015 and 2018 . Combined analyses of the collected data from the two experiments will push the precision even further.

The third LHC run starts in 2022 and the LHC Upgrade, the High-Luminosity LHC, is scheduled to go 
into operation in 2027. The plan is to increase the recorded data set by about a factor of 20 , allowing the hunt for even rarer processes and more precise measurements.

One goal will be the measurement of the Higgs boson couplings to other particles with precisions up to a few percent [13]. Another major goal will be to measure the self-interaction of the Higgs boson, which is sensitive to the energy potential of the scalar field, and therefore another important test of the Higgs mechanism and the structure of the vacuum.

The highlighted studies, both at the LHC and at future colliders, all have the purpose of stress-testing the Higgs sector of the Standard Model, as we are looking for tiny hints that could answer some of the big questions about our universe.

\section{About the author}

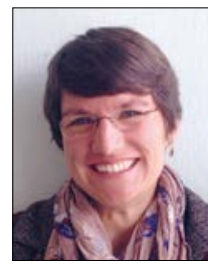

Sarah Heim is a Helmholtz Young Investigator Group leader at DESY in Hamburg, Germany. She investigates the properties of the Higgs boson and searches for dark matter using data from the LHC. She is also involved in the upgrade of the ATLAS tracking detector for the High-Luminosity LHC.

\section{Further reading}

In general, the physics briefings of the ATLAS and CMS experiments give a good overview over recent results: https://atlas.cern/updates/physics-briefing https://cms.cern/tags/physics-briefing

\section{References}

[1] CMS Collaboration, Phys. Lett. B 805, 135425 (2020)

[2] ATLAS Collaboration, Measurement of the Higgs boson mass in the $H \rightarrow Z Z^{*} \rightarrow 4 \ell$ decay channel with $\sqrt{ } s=13 \mathrm{TeV} p p$ collisions using the ATLAS detector at the LHC, ATLAS-CONF-2020-005

[3] ATLAS Collaboration, Phys. Lett. B 812, 135980 (2021)

[4] CMS Collaboration, JHEP 01, 148 (2021)

[5] ATLAS Collaboration, A combination of measurements of Higgs boson production and decay using up to $139 \mathrm{fb}^{-1}$ of protonproton collision data at $\sqrt{ } s=13$ TeV collected with the ATLAS experiment, ATLAS-CONF-2020-027

[6] CMS Collaboration, Combined Higgs boson production and decay measurements with up to $137 \mathrm{fb}^{-1}$ of proton-proton collision data at $\sqrt{ } s=13 \mathrm{TeV}, \mathrm{CMS}-\mathrm{PAS}-\mathrm{HIG}-19-005$

[7] ATLAS Collaboration, Combination of searches for invisible Higgs boson decays with the ATLAS experiment, ATLAS-CONF-2020-052

[8] CMS Collaboration, Phys. Lett. B 793, 520 (2019)

[9] ATLAS Collaboration, Phys. Rev. Lett. 125, 061802 (2020)

[10] CMS Collaboration, Phys. Rev. Lett. 125, 061801 (2020)

[11] CMS Collaboration, Analysis of the CP structure of the Yukawa coupling between the Higgs boson and $\tau$ leptons in protonproton collisions at $\sqrt{ } s=13 \mathrm{TeV}, \mathrm{CMS}-\mathrm{PAS}-\mathrm{HIG}-20-006$

[12] ATLAS Collaboration, Interpretations of the combined measurement of Higgs boson production and decay, ATLAS-CONF-2020-053

[13] LHC Higgs working group 2, Higgs Physics at the HL-LHC and HE-LHC, CERN-LPCC-2018-04

\section{n CAEN Electronic Instrumentation}

\section{DIGITAL SPECTROSCOPY}

\section{HEXAGON MCA \& Quantus Software}

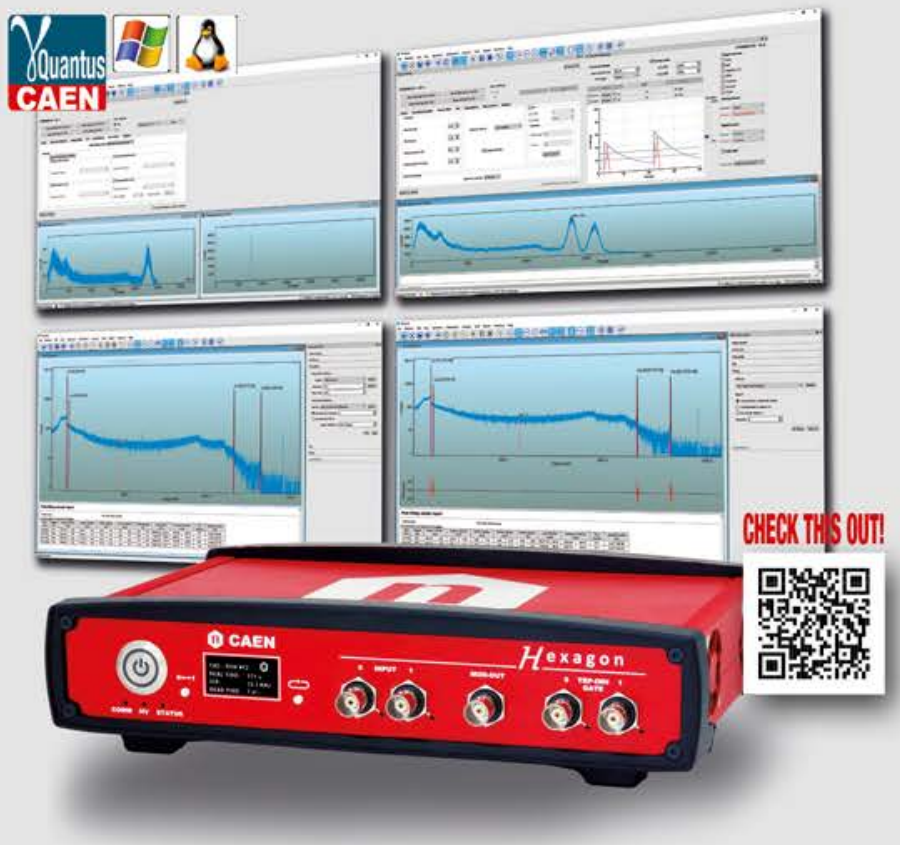

\section{Quantus - Quantitative Spectrometry Software}

- Compliant with ISO11929 standard

- Spectrum analysis through a powerful collection of calculation engines:

- ROI computations

- Peak search and Peak fitting algorithms

- Multiplet deconvolution and automatic ROI definition

- Energy, FWHM and Efficiency calibration

- Activity calculation algorithms

- Radionuclide identification and quantification

- Advanced HTML reports

\section{HEXAGON - Digital MCA Multichannel Analyzer}

- Single and Dual 32k Digital MCA \& Pulse Processor

- Pulse Height Analysis, Advanced input channel correlation (coinc/anticoinc), Time-stamped Lists, SCA and MCS

- Ideally suited for HPGe, CZT, Silicon, and Scintillation detectors

- Supports Resistive Feedback and TRP as well as PMT anode signals 